\title{
Desarrollo e implementación de un modelo de auditoría médica basado en el aseguramiento de la calidad y la supervisión participativa en Ecuador
}

\author{
Development and implementation of a medical audit model based on quality assurance and \\ participatory supervision in Ecuador
Desenvolvimento e implementação do modelo de auditoria médica baseada na garantia da qualidade e monitoramento participativo no Equador

Fausto Gady Torres Toala ${ }^{a}$

\section{Resumen}

Objetivos: Este estudio es una descripción del desarrollo de una estrategia de Auditoría Médica basada en el Aseguramiento de la Calidad de la prestación utilizando herramientas de supervisión participativa en un intento de generar cooperación entre el auditor y el auditado en servicios de salud del primer nivel de atención del Ministerio de Salud Pública de Ecuador. Métodos: Se desarrolló y se implementó una metodología la cual se probó en el terreno, basados en una descripción de los resultados (corte transversal). Resultados: Al inicio del proceso, las puntuaciones de pertinencia clínica, de manera global, no superaban el $45 \%$ y respecto al riesgo legal no superan el $65 \%$. Progresivamente se observa un aumento de la puntuación, adicionalmente se obtuvieron resultados propios de la percepción de este proceso por parte de los auditados, teniendo resistencia en menos del $5 \%$ de los casos, quizás debido al carácter participativo del proceso. Conclusión: El proceso de auditoría Médica es indispensable para la identificación de problemas, pero también es necesario que sea un proceso activo con intervención y resolución de los mismos. Auditoría Médica identifica problemas ocultos para los tomadores de decisiones y disminuye la resistencia en la inclusión de estrategias de mejoramiento de la calidad.

\section{Abstract}

Objectives: This study is a description of the development of a medical audit strategy based on quality provision assurance using tools of participatory supervision in an attempt to generate cooperation between the auditor and the auditee in health services of the first level of Primary Attention to Health Care of the Ministry of Public Health of Ecuador. Methods: A methodology was developed, implemented and field tested, based on a description of the results (cross-section study). Results: At the beginning of the process, the clinical relevant scores, in general, did not exceed $45 \%$ and in relation to legal risks they did not exceed 65\%. Progressively, an increase in the score was observed. Additionally, results obtained from the auditees' perception of this process were obtained, with less resistance in less than $5 \%$ of the cases, perhaps due to the participatory nature of the process. Conclusion: The medical audit process is essential for the identification of problems, but it is also necessary to be an active process with their intervention and resolution. The Medical Audit identifies hidden problems for decision-makers and decreases the resistance to the inclusion of strategies for quality improvement.
Palabras clave:

Auditoría Médica

Círculos de Calidad

Aseguramiento de la Calidad de Atención de Salud

Keywords:

Medical Audit

Quality Circles

Quality Assurance in Health Care
Cómo citar: Torres FG. Desarrollo e implementación de un modelo de auditoria medica basado en garantía de calidad y supervisión participativa en Ecuador. Rev Bras Med Fam Comunidade. 2017;12(39):1-11. http://dx.doi.org/10.5712/rbmfc12(39)1417
Financiación: ninguna declarada. Aprobación ética: no aplicable.

Conflicto de intereses: ninguno declarado. Procedencia y revisión por pares: revisado por pares. Recibido el: 08/11/2016.

Aceptado el: 08/05/2017. 


\section{Resumo}

Objetivos: Este estudo é uma descrição do desenvolvimento de uma estratégia de auditoria médica baseada na garantia da provisão de qualidade usando ferramentas de monitoramento participativo em uma tentativa de gerar cooperação entre o auditor e o auditado no primeiro nível de serviços de Atenção Primária de Saúde do Ministério da Saúde Pública do Equador. Métodos: Desenvolvimento e implementação de uma metodologia, testada em campo, baseada em uma descrição dos resultados (estudo de corte transversal). Resultados: No início do processo, a pontuação de relevância clínica, em geral, não superava $45 \%$ e os riscos legais não excediam $65 \%$. Progressivamente, observa-se um aumento na pontuação; adicionalmente, obtiveram-se resultados próprios de percepção deste processo por parte dos auditados, tendo menos resistência em menos de $5 \%$ dos casos, talvez devido ao caráter participativo do processo. Conclusão: O processo de auditoria médica é essencial para a identificação de problemas, mas também precisa ser um proceso ativo, com intervenção e resolução dos mesmos. A Auditoria Médica identifica problemas ocultos para os tomadores de decisão e diminui a resistência à inclusão de estratégias para a melhoria da qualidade.
Palavras-chave:

Auditoria Médica

Participação nas Decisões

Garantia da Qualidade

dos Cuidados de Saúde

\section{Introducción}

El Proyecto Aseguramiento Universal en Salud (AUS) fue una política pública que se aplicó en Ecuador, con el cambio de gobierno en el 2007 el AUS fue dejado en segundo plano, sin embargo, aportó múltiples experiencias que se han desarrollado hasta la actualidad, fue el escenario ideal junto a la voluntad política para crear la estrategia de Auditoría Médica y probarla a gran escala.

El proceso de diseño se caracterizó por la construcción de un marco conceptual de referencia que fue la base para la estrategia, de manera tal que no generó resistencias y además no fue incompatible con la realidad local. Este esfuerzo se realizó con un equipo con experiencia local en auditoría y se reflejó en el manual de auditoría del AUS, publicado en el año 2006, que fue un documento que recopiló la estrategia y los resultados de la implementación inicial de la Auditoría médica.

Otro aspecto clave, fue la validación de los procesos al ser comparados con experiencias internacionales, lo que le dio mayor fuerza técnica y corrigió ciertos potenciales errores que podrían ocurrir en la implementación. Podemos decir que el proceso de auditoría creado en el MSP (Ministerio de Salud Pública del Ecuador) que se inició desde el 2006, es un proceso creado para el contexto sanitario ecuatoriano sin perder criterios técnicos de calidad, que tomó aspectos teóricos y operativos de otros procesos internacionales y los adaptó de acuerdo a las necesidades del sistema de salud a ser auditado. El desarrollo del proceso de auditoría, además de ser contextualizado, se basa en experiencias de supervisión participativa y de manejo de organizaciones eficientes. ${ }^{1-4} \mathrm{El}$ proceso de Auditoría diseñado, contó con la experiencia de un equipo en una organización ad hoc ${ }^{5}$ que tomó los elementos más relevantes de varias experiencias locales, regionales y globales, obteniendo como resultado una estrategia de control de la calidad de la prestación eficiente y efectiva, que disminuye los conflictos en su implementación. . $^{6-10}$

\section{Métodos}

Es un estudio exploratorio para el desarrollo de la metodología y con un componente de corte transversal para evidenciar los resultados de la implementación.

Inicialmente se describe el desarrollo de una metodología adaptada de la supervisión participativa, modelándola con principios de aseguramiento de la calidad, mejora continua, no punitiva, construcción de competencias y excelencia, ${ }^{3,11-16}$ posteriormente se presentan resultados de la primera intervención y se hace una reflexión de las aplicaciones posteriores. 
Todo el proceso de diseño fue una estrategia de gestión, ante el problema de tener una incertidumbre de cuál sería la calidad de la prestación en salud como producto final en un ambiente de capitación como forma de pago en el AUS. Esto desencadenó de manera lógica e inmediata la conformación de un grupo de trabajo conformado por profesionales en Salud Pública, Auditoría Médica y Medicina Familiar, con lo que se esperaba cubrir técnicamente las dudas que se vayan planteando en el proceso.

El grupo conformado, tenía una estructura adhoc, es decir que tiene una estructura flexible, diferente que una estructura burocrática disfuncional a pesar de estar inmersa en ella. Las líneas de poder son distribuidas selectivamente de manera horizontal y/o vertical dependiendo de las necesidades de producción. Desaparece el ápice estratégico ya que se conforma por unidades de expertos que se coordinan entre sí por adaptación mutua, por lo que el flujo de información y la calidad de discusión, innovación y retroalimentación fue muy alta. Adicionalmente a este equipo se contaba con expertos en otras materias que daban ideas de mejora del proyecto.

El poder conformar este equipo multidisciplinario e interinstitucional, con alto nivel técnico, logró que el clima organizacional sea agradable y permita el proceso de diseño teórico de lo que sería un proceso innovador en cuestión de aseguramiento de la calidad de la prestación, el producto final de este grupo de trabajo sería el manual de procesos del área de control, herramienta indispensable para la operativización de dicha área en el AUS (Figura 1).

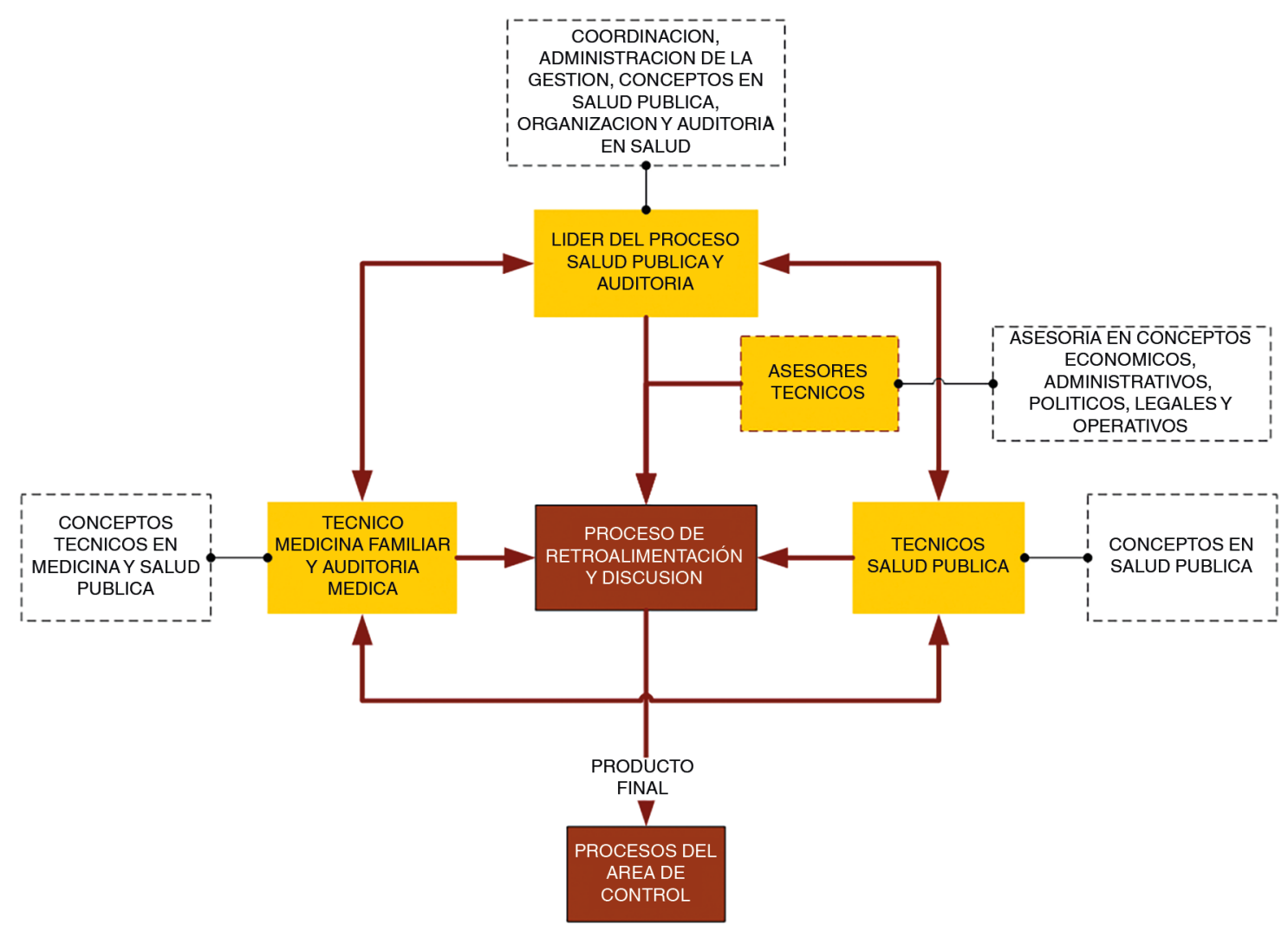

Figura 1. Modelo de organización adhoc para el desarrollo del área de control. 
Dentro del marco conceptual de referencia, el principal producto fue la redefinición del concepto clásico de la auditoría médica. Cambiar el imaginario médico de un proceso de control estricto y convertirlo en un proceso cooperativo de mejoramiento de la calidad y en una herramienta para la toma de decisiones del gerente.

La Auditoría Médica como se la plantea en este documento interviene en todos los aspectos de la gestión: ${ }^{11,12,17-19}$

1. Macrogestión: Auditoría médica es una herramienta de la generación de la política sanitaria o de las estrategias más locales para mejorar los servicios de salud, identifica las debilidades del sistema y propone alternativas participativas de cambio.

2. Mesogestión: En el proceso administrativo de un servicio de salud, es clave determinar los niveles de sobre o sub prestación para determinar estrategias de contención de costos, también es importante conocer el nivel de calidad de la asistencia para determinar procesos de capacitación y así mejorar el impacto sanitario en la comunidad.

3. Microgestión: a nivel del proceso de relación del personal de salud con el usuario, al identificar errores en terreno que causen errores clínicos y al proponer soluciones in situ, se convierte en un proceso de mejoramiento continuo de la calidad.

Todos los procesos diseñados en la Auditoría Médica del AUS, tienen instrumentos ponderados los cuales están diseñados para producir variables y así alimentar una tabla de datos. Los datos que se producen pueden producir información, esta información es de utilidad para el personal operativo (es una referencia de la calidad de la asistencia) y para el gerente o mando medio (datos agregados de eficiencia y efectividad) útiles en la toma de decisiones para mejorar la calidad.

\section{Auditoría Médica como un proceso participativo}

El proceso fue diseñado para ser participativo, en el concepto clásico, la participación se reducía a las apelaciones que no son más que reclamaciones ante la decisión del auditor. En nuestro proceso antes que ocurra una decisión del auditor existe el proceso participativo (Figura 2), el subproceso de auditoría de terreno es el encargado de generar la participación de los trabajadores del servicio de salud al proponer su estrategia de supervisión participativa.

Ei proceso de Auditoría Médica diseñado, garantiza la participación mediante las siguientes estrategias:

- Entrega transparente de información

- Diálogo previo a la auditoría

- Negociación de las estrategias de cambio

- Cooperación en procesos de mejoramiento de la calidad

- Apelaciones en consenso técnico

Organización en la que debe funcionar el proceso de Auditoría Médica y talento humano

Posteriormente el proceso se lleva a cabo por profesionales médicos que se forman en la metodología y se aplica en diversos ambientes públicos y privados. 


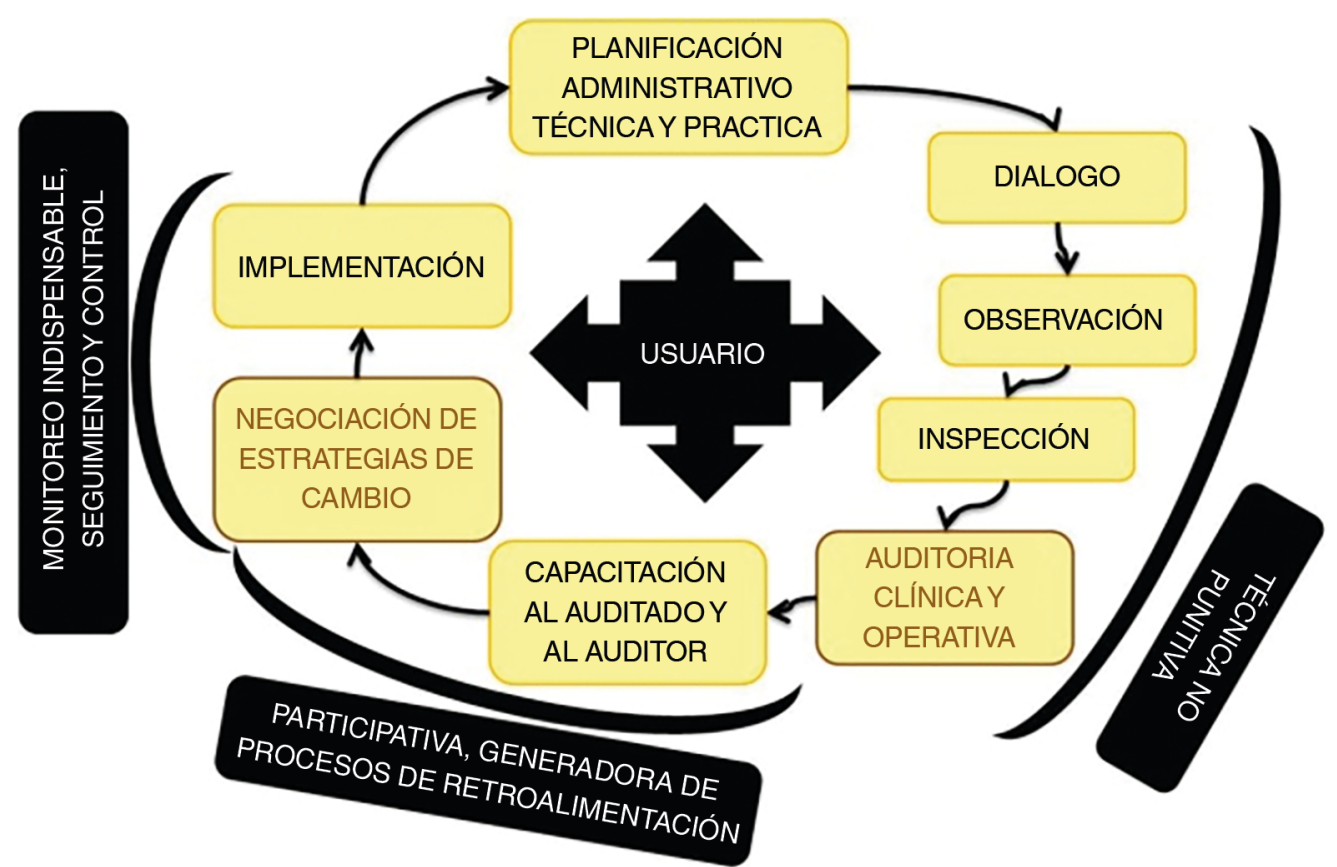

Figura 2. Modelo de Auditoría desarrollado en el AUS.

Para el diseño de la organización denominada Auditoría Médica, se recurrió a la propuesta de Mintzberg, en función de lo cual establecemos una organización que mantiene el ápice gerencial y un mando medio, el núcleo operativo es el motor del proceso, con una tecnoestructura conformada por un proceso de calidad y un personal de apoyo no tan prominente debido a la automatización de los procesos. ${ }^{1,2}$

La coordinación del trabajo en esta organización será mediante la adaptación mutua, estandarización de las normas y del proceso del trabajo, con una configuración innovadora (adhocracia). ${ }^{5}$ Con esto logramos que los integrantes del equipo logren complementarse, se evite la verticalidad y además los impulsos vayan hacia el empoderamiento del mejoramiento continuo de la calidad de la salud tomando en cuenta sus determinantes sociales (Figura 3); esta forma organizativa horizontal se mantuvo en el posterior desarrollo del instrumento, dando excelentes resultados.

En Ecuador, existe la formación de Auditoría Médica a través de certificaciones, por ejemplo, el Taller de Auditoría Médica de instituciones privadas como Makroscopio y la Universidad San Francisco de Quito, en el 2016 y 2017 están en proceso de aprobación estudios de posgrado relacionados al tema. En el momento de la auditoría, los auditores se formaron en el terreno, el líder del Equipo (el autor), diseñó y aplicó el proceso de entrenamiento.

Metodología y marco conceptual de referencia

El modelo de referencia de este subproceso fue la Supervisión Participativa ${ }^{20-23}$ y la Auditoría Concurrente o de terreno clásica. Al referirnos al concepto clásico, hablamos del modelo de auditoría más utilizado (Figura 4). 


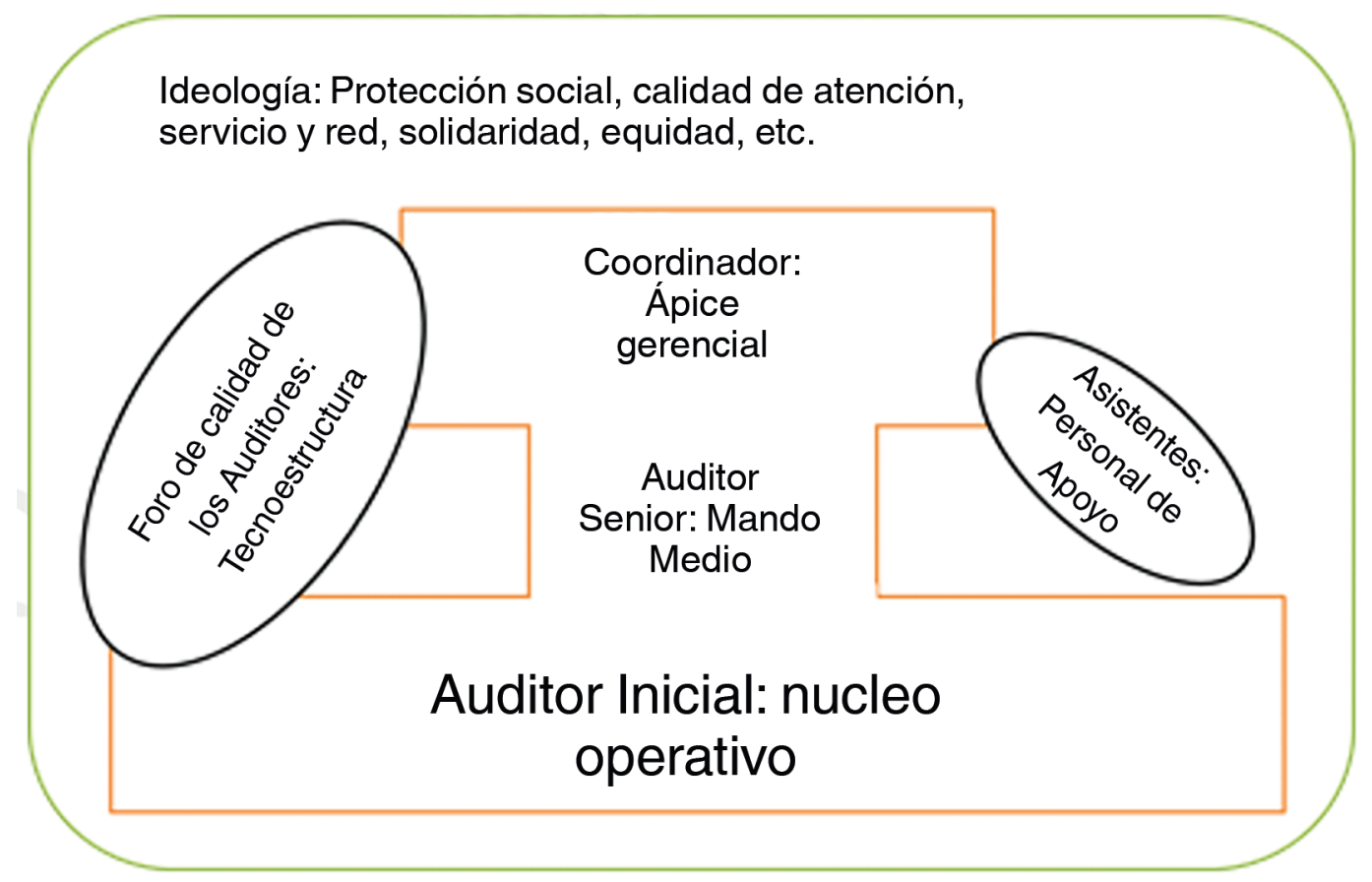

Figura 3. Modelo de organización de Auditoría Médica.

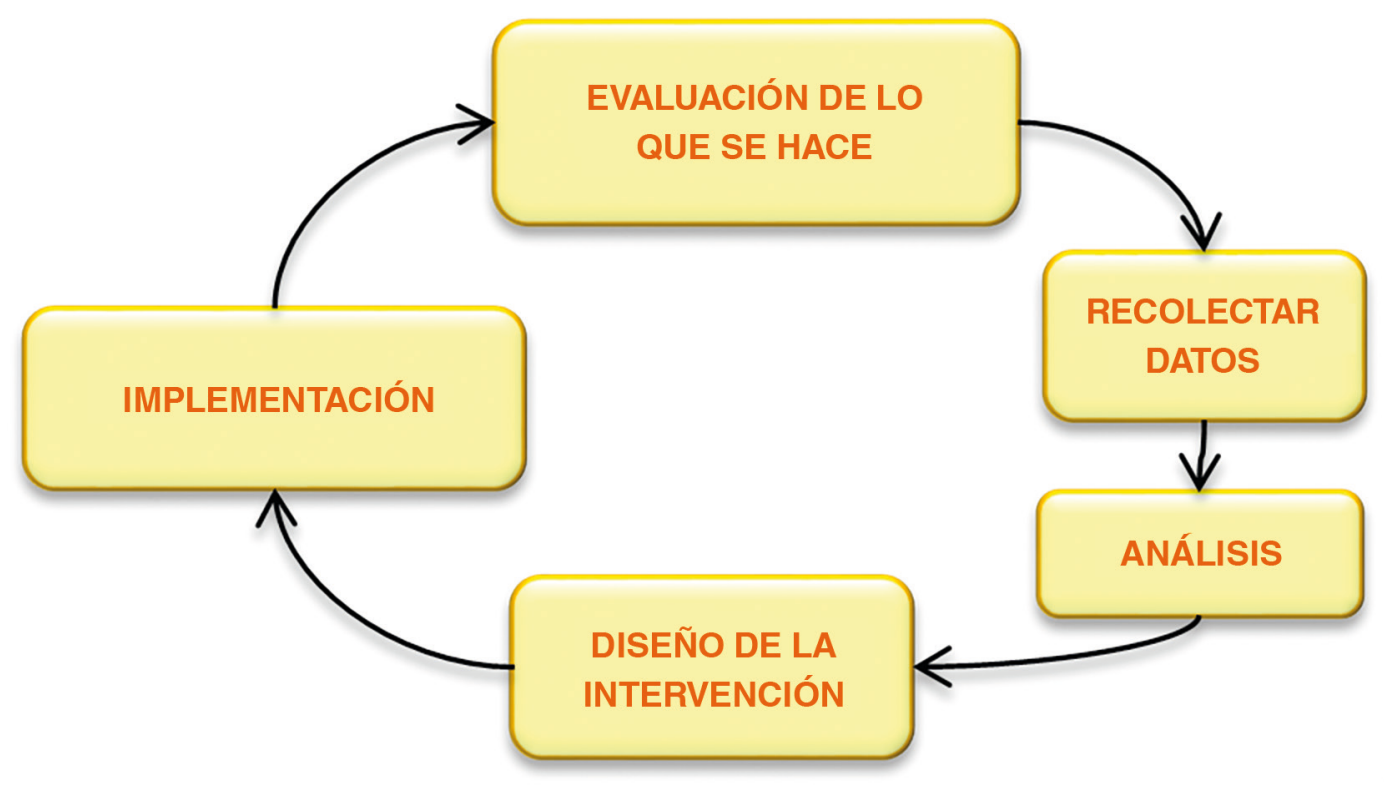

Figura 4. Modelo clásico de Auditoría Médica.

En dicho modelo, se describe un proceso circular de control, en el cual no hay niveles de participación adecuados, esto puede causar potencialmente resistencia del personal de salud, la resistencia se visualiza como conflictos o curvas planas en el mejoramiento de la calidad. El modelo que construimos se constituye en el pilar fundamental de todo el proceso de auditoría desarrollado, debido a que es el único que tiene en cuenta la participación mediante procesos de negociación además de la capacitación in situ y deja abierta la posibilidad de cooperación técnica del auditor hacia el auditado. La auditoría de terreno se construye como el proceso de 
prevención de los errores del prestador en la entrega de sus servicios de salud, es decir se realiza cuando la prestación está ocurriendo (por lo que se advierten los errores antes de la finalización del episodio) o cuando la prestación ya ocurrió (entonces se la toma como ejemplo para evitar errores en prestaciones similares).

\section{Resultados}

\section{Guía de campo de la Auditoría de terreno}

Para nosotros la guía de campo es el primer resultado exitoso, es un instrumento eficiente ya que la misma guía de campo actúa como el informe individual de auditoría, ya que se registran en ella todos los problemas encontrados y las recomendaciones, así como las posibles apelaciones, además es un documento que evidencia la auditoría y es firmado por el auditor y un representante de los auditados. El instrumento fue desarrollado a partir de una base teórica política en el Aseguramiento Universal en Salud, se ha validado en el terreno y ha demostrado ser eficiente y efectivo para evaluar la historia clínica.

Hay que aclarar que este es un formulario inicial y que conforme la calidad de la prestación aumente, este formulario se hará más complejo hasta llegar a niveles máximos de calidad. Si se inician con los valores ideales, esto genera problemas de desmotivación en la cual el prestador no los alcanzará a corto ni a mediano plazo sino a largo plazo.

Esta guía de campo tiene 2 partes que dan una puntuación, la pertinencia clínica y el riesgo legal. Cada una de ellas consta de varios ítems, los cuales serán ponderados por la importancia que tengan. Al final se otorgará un peso a la parte clínica y otro al riesgo legal que en conjunto será la puntuación global de la auditoría de terreno realizada. La importancia de la ponderación radica en que el auditado tiene un documento objetivo de su evaluación y conocerá en qué específicamente fue el error. Por lo tanto, tiene un referente para el proceso continuo de control.

La pertinencia clínica constaba de varias dimensiones: referencia y contrarreferencia, racionalidad de la atención curativa y preventiva y uso de recursos (medicamentos, exámenes de laboratorio y de imágenes); el riesgo legal a su vez, estaba conformado por: registro adecuado de identificación de la historia clínica, identificación del médico tratante, legibilidad, inclusión de documentación obligatoria. Vale la pena recalcar que este instrumento es dinámico y va adquiriendo o complejizando dimensiones de acuerdo a la evolución del mejoramiento de la calidad.

\section{Aspectos actitudinales de los auditados}

Se realiza de manera semestral, con un enfoque cualitativo, es un análisis básico que incluye:

1. Estructura básica del lenguaje

2. Análisis narrativo

En la primera evaluación, los resultados son sorprendentes, se encuentra un discurso explícito que se basa en excusas por tiempo y al mismo tiempo en defensa de la institución, pero otro discurso implícito de abandono, el personal operativo lo percibe debido a la ausencia de capacitación o de acercamiento con los mandos gerenciales. Este análisis lo aprovecha la Auditoría Médica para convertirse en el intermediario y llevar la capacitación a la unidad operativa (Figura 5). 


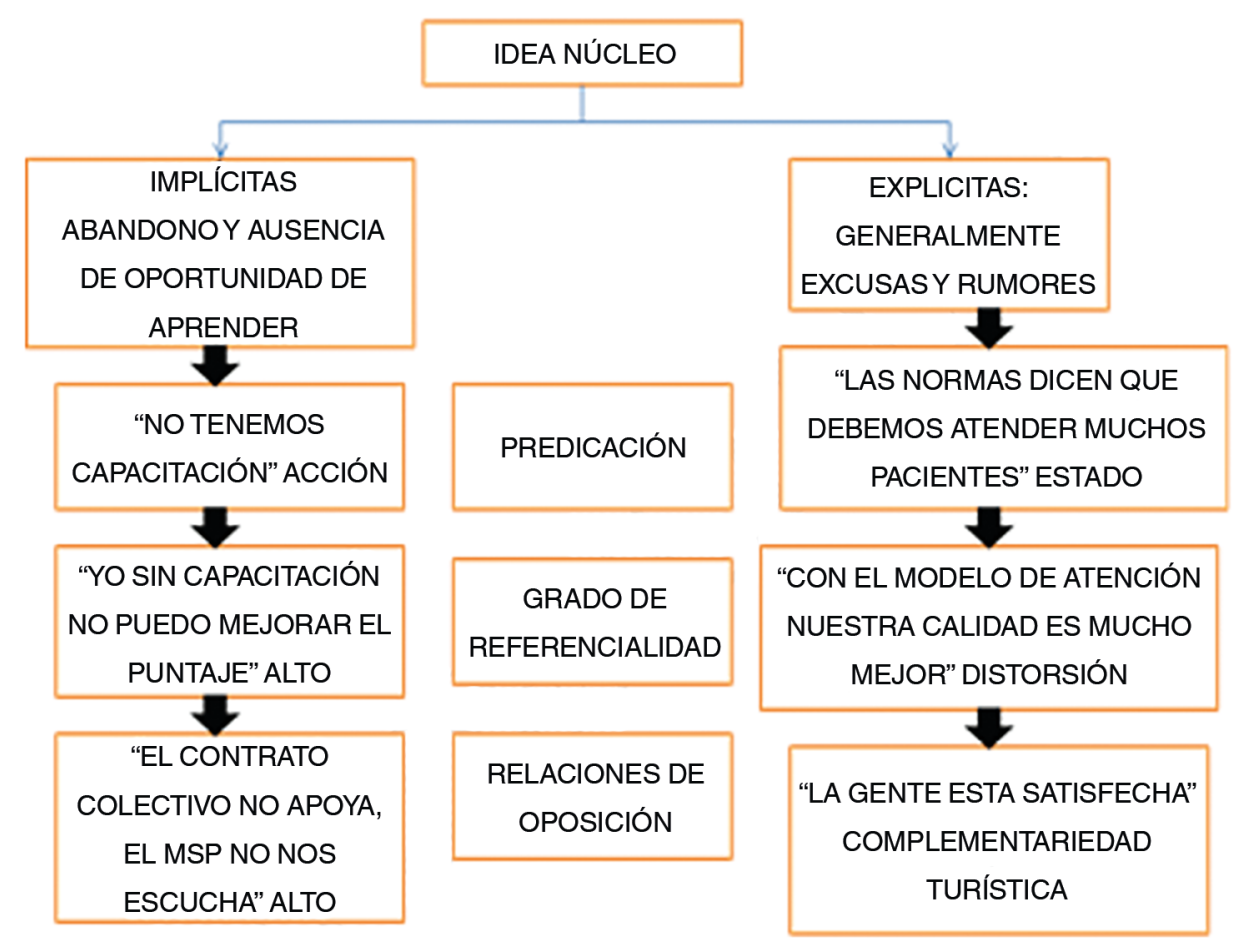

Figura 5. Evaluación cualitativa del Auditado.

\section{Resultados de la auditoría de servicios de salud}

Se auditaron 1920 casos en un periodo de 10 meses, se inicia con valores muy bajos de pertinencia clínica, de tan solo $42 \%$, es decir que solo 4 de cada 10 historias tenían una calidad de la atención clínica aceptable, las otras tenían algún tipo de error, sin embargo, la intervención de auditoría logra que en 10 meses se mejore y se culmine con un 64\%, la evaluación de riesgo legal única en 63\% y culmina en 83\% (Figura 6).

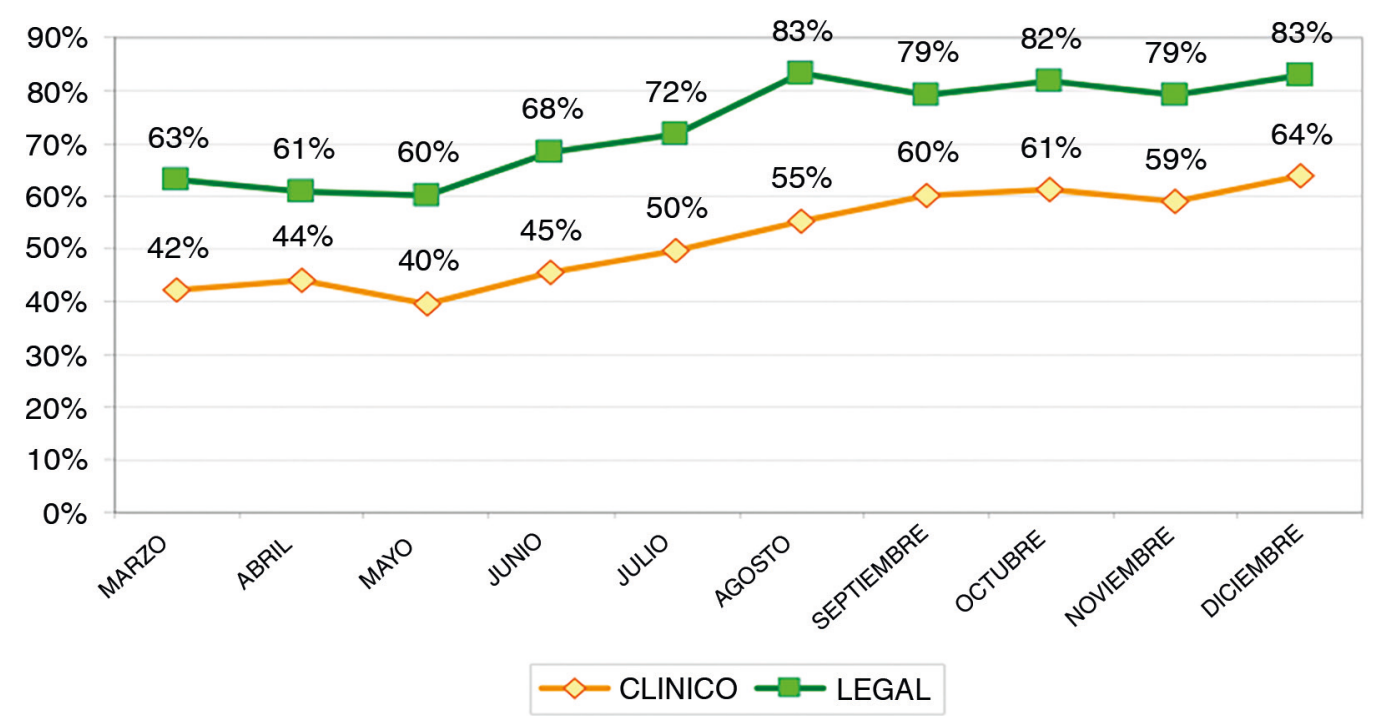

Figura 6. Evolución de la evaluación de Auditoría Clínica. 


\section{Creación de espacios de reflexión auditor auditado}

Es un proceso que se dio de manera espontánea en el proceso, se crearon espacios de discusión auditor auditado, fuera de la auditoría. Estos espacios fueron iniciados por el prestador mismo y variaron en forma y estrategia de comunicación, pero su objetivo fue el mismo: entender el proceso de auditoría, resolver apelaciones y buscar estrategias para mejorar.

\section{Creación Evolución y sostenibilidad de la estrategia}

La inestabilidad política y los cambios en el país, no permitieron que se consolide el proceso de auditoría médica en su totalidad, sin embargo, fue la base para futuros procesos que se mantienen actualmente. Esta estrategia fue desarrollada y continuada en instituciones del sector privado y también en casos de alta complejidad en el sector público. Actualmente la estrategia sigue vigente y se sigue aplicando en el contexto ecuatoriano.

\section{Discusión}

Los procesos de Auditoría Médica en Ecuador se han desarrollado en algunos subsistemas públicos y privados, sin embargo, era muy débil en el Ministerio de Salud Pública quien además de ser el rector del Sistema Nacional de Salud también es un proveedor de servicios de salud, su experiencia comienza con la propuesta AUS y se ha desarrollado de manera permanente.

Varios autores han coincidido que las intervenciones basadas en retroalimentación y que involucren actividades de docencia han dado resultados pequeños a moderados, ${ }^{4,9,24-28}$ incluso a nivel de terapia profesional a pacientes en salud mental ha sido documentado, lo cual es coherente con nuestro modelo, sin embargo, hemos logrado resultados importantes en poco tiempo, quizás debido al modelo de gestión que manejamos.

Paton ha demostrado que el simple hecho de tener datos e indicadores no ha generado mejoras sustanciales en calidad, ${ }^{10}$ Zhele propone que los cambios deben incidir en el comportamiento de los profesionales directamente ${ }^{29}$ lo cual es coherente con la preocupación de varios investigadores sobre la no utilización de Guías Clínicas, ${ }^{30-32}$ nuestra metodología tuvo en cuenta todos los enfoque y generó estrategias para la disminución de resistencia al cambio, la retroalimentación dentro de un ambiente de supervisión participativa.

Este proceso ha demostrado ser eficiente, porque el número de auditores participantes e instrumentos automatizados fueron capaces de realizar una auditoría de calidad en la capital del Ecuador.

Los obstáculos encontrados en esta intervención fueron:

- Falta de protocolos o guías de práctica clínica

- La falta de oportunidades para acceder a la capacitación formal en la salud y la educación médica continua

- La falta de capacitación de los auditores en el país 


\section{Conclusión}

La Auditoría medica ha demostrado ser un proceso eficiente y efectivo en el mejoramiento de la calidad de la prestación de los servicios médicos, las intervenciones de este tipo deben ser construidas de manera contextualizada y además deben ser participativas y empáticas, teniendo en cuenta las barreras de los profesionales de la salud para poder ejercer con calidad su actividad, no debe ser un proceso punitivo sino colaborativo.

La Auditoría médica es una fuente importante de información para la construcción de políticas sanitarias y para el desenmascaramiento de problemas en el nivel operativo.

\section{Referencias}

1. Mintzberg H, Quinn JB, Voyer J. El proceso estratégico: Conceptos, contextos y casos. Naucalpan de Juárez: Prentice-Hall Hispanoamericana; 1997.

2. Mintzberg H. La estructuración de las organizaciones. Esplugues de Llobregat Barcelona: Ariel; 2012.

3. Sedano AM. La supervisión educativa. Bordón Rev Pedagog. 1991;43(2):177-86.

4. Holloway EL, Neufeldt SA. Supervision: its contributions to treatment efficacy. J Consult Clin Psychol. 1995;63(2):207-13. DOI: http://dx.doi.org/10.1037/0022-006X.63.2.207

5. Mintzberg H, McHugh A. Strategy Formation in an Adhocracy. Adm Sci Q. 1985;30(2):160-97. DOI: http://dx.doi. org/10.2307/2393104

6. Dewulf A, Gray B, Putnam L, Lewicki R, Aarts N, Bouwen R, et al. Disentangling approaches to framing in conflict and negotiation research: A meta-paradigmatic perspective. Hum Relat. 2009;62(2):155-93. DOI: http://dx.doi. org/10.1177/0018726708100356

7. Oetzel JG, Ting-Toomey S. Face Concerns in Interpersonal Conflict: A Cross-Cultural Empirical Test of the Face Negotiation Theory. Commun Res. 2003;30(6):599-624. DOI: http://dx. doi.org/10.1177/0093650203257841

8. Lewicki RJ, Weiss SE, Lewin D. Models of conflict, negotiation and third party intervention: A review and synthesis. J Organ Behav. 1992;13(3):209-52. DOI: http://dx.doi.org/10.1002/job.4030130303

9. Jamtvedt G, Young JM, Kristoffersen DT, O'Brien MA, Oxman AD. Does telling people what they have been doing change what they do? A systematic review of the effects of audit and feedback. Qual Saf Health Care. 2006;15(6):433-6. DOI: http://dx.doi.org/10.1136/qshc.2006.018549

10. Paton JY, Ranmal R, Dudley J; RCPCH Clinical Standards Committee. Clinical audit: still an important tool for improving healthcare. Arch Dis Child Educ Pract Ed. 2015;100(2):83-8. DOI: http://dx.doi.org/10.1136/archdischild-2013-305194

11. Neuspiel DR, Stubbs EH, Liggin L. Improving reporting of outpatient pediatric medical errors. Pediatrics. 2011;128(6):e160813. DOI: http://dx.doi.org/10.1542/peds.2011-0477

12. Hysong SJ, Best RG, Pugh JA. Audit and feedback and clinical practice guideline adherence: making feedback actionable. Implement Sci. 2006;1:9. DOI: http://dx.doi.org/10.1186/1748-5908-1-9

13. Runyon KE. Some interactions between personality variables and management styles. J Appl Psychol. 1973;57(3):288-94. DOI: http://dx.doi.org/10.1037/h0034723

14. Spector PE. Behavior in organizations as a function of employee's locus of control. Psychol Bull. 1982;91(3):482-97. DOI: http://dx.doi.org/10.1037/0033-2909.91.3.482

15. Mitchell TR, Smyser CM, Weed SE. Locus of Control: Supervision and Work Satisfaction. Acad Manage J. 1975;18(3):62331. DOI: http://dx.doi.org/10.2307/255692

16. Katz FM, Snow R. Evaluación del rendimiento de los trabajadores de la salud: Manual de formación y supervisión. Cuad Salud Púbica OMS [Internet]. 1981. [citado 2016 Nov 22]. Disponible en: http://bases.bireme.br/cgi-bin/wxislind.exe/iah/ online/?IsisScript=iah/iah. xis\&src=google\&base=PAHO\&lang=p\&nextAction=Ink\&exprSearch=1619\&indexSearch=ID 
17. Hysong DS. Meta-analysis: audit and feedback features impact effectiveness on care quality. Med Care. 2009;47(3):35663. DOI: http://dx.doi.org/10.1097/MLR.0b013e3181893f6b

18. Neuspiel DR, Stubbs EH. Patient safety in ambulatory care. Pediatr Clin North Am. 2012;59(6):1341-54. DOI: http://dx.doi. org/10.1016/j.pcl.2012.08.006

19. Shumaker SA, Ockene JK, Riekert KA, eds. The Handbook of Health Behavior Change. 3rd Ed. New York: Springer;2008.856 p.

20. Gas HGA, González BM. Calidad y liderazgo en medicina. Rev CONAMED [Internet]. el 28 de abril de 2015. [citado 2017 Abr 27];18(4):172-82. Disponible en: http://www.dgdi-conamed.salud.gob.mx/ojs-conamed/index.php/revconamed/article/view/127

21. Jimenez Trespalacios E. Diseño del programa de auditoria para el mejoramiento de la calidad de la atencion en salud de la unidad funcional de hospitalizacion de la policlinica Ismael Dario Rincon, en la regional de salud del Magdalena Medio de Ecopetrol s.a. en Barrancabermeja. [Internet] [Thesis]. 2012 [citado 2017 Abr 27]. Disponible en: http://bdigital.ces.edu. co:8080/repositorio/handle/10946/305

22. Jiménez Cangas L, Báez Dueñas RM, Pérez Maza B, Reyes Alvarez I. Metodología para la evaluación de la calidad en instituciones de atención primaria de salud. Rev Cubana Salud Pública. 1996;22(1):15-6.

23. Isermann R. Supervision, fault-detection and fault-diagnosis methods - An introduction. Control Eng Pract. 1997;5(5):63952. DOI: http://dx.doi.org/10.1016/S0967-0661(97)00046-4

24. O'Brien MA, Rogers S, Jamtvedt G, Oxman AD, Odgaard-Jensen J, Kristoffersen DT, et al. Educational outreach visits: effects on professional practice and health care outcomes. Cochrane Database Syst Rev. 2007;(4):CD000409.

25. Ivers N, Jamtvedt G, Flottorp S, Young JM, Odgaard-Jensen J, French SD, et al. Audit and feedback: effects on professional practice and healthcare outcomes. Cochrane Database Syst Rev. 2012;(6):CD000259.

26. Forsetlund L, Bjørndal A, Rashidian A, Jamtvedt G, O'Brien MA, Wolf F, et al. Continuing education meetings and workshops: effects on professional practice and health care outcomes. Cochrane Database Syst Rev. 2009;(2):CD003030.

27. Jamtvedt G, Young JM, Kristoffersen DT, O'Brien MA, Oxman AD. Audit and feedback: effects on professional practice and health care outcomes. Cochrane Database Syst Rev. 2006;(2):CD000259.

28. Watkins Jr CE. Does Psychotherapy Supervision Contribute to Patient Outcomes? Considering Thirty Years of Research. Clin Superv. 2011;30(2):235-56. DOI: http://dx.doi.org/10.1080/07325223.2011.619417

29. Zhelev Z, Abbott R, Rogers M, Fleming S, Patterson A, Hamilton WT, et al. Effectiveness of interventions to reduce ordering of thyroid function tests: a systematic review. BMJ Open. 2016;6(6):e010065. DOI: http://dx.doi.org/10.1136/ bmjopen-2015-010065

30. González-Juanatey JR, Alegría-Ezquerra E, Aznar-Costa J, Bertomeu-Martínez V, Franch-Nadal J, Palma-Gámiz JL. Conocimiento y aplicación de las guías de práctica clínica sobre riesgo cardiovascular en las consultas generales y especializadas. Rev Esp Cardiol. 2006;59(8):801-6. DOI: http://dx.doi.org/10.1157/13091884

31. Letelier SML, Moore P. La medicina basada en evidencia:Visión después de una década. Rev Méd Chile. 2003;131(8):939-46.

32. Rello J, Lorente C, Bodí M, Diaz E, Ricart M, Kollef MH. Why do physicians not follow evidence-based guidelines for preventing ventilator-associated pneumonia?: a survey based on the opinions of an international panel of intensivists. Chest. 2002;122(2):656-61. DOI: http://dx.doi.org/10.1378/chest.122.2.656

a Makroscopio Servicios de Salud. Ecuador. gtorres@makroscopio.com (Autor correspondiente) 